\title{
The TNF-family cytokine TL1A promotes allergic immunopathology through group 2 innate lymphoid cells
}

\author{
F Meylan ${ }^{1}$, ET Hawley ${ }^{1}$, L Barron ${ }^{2}$, $\mathrm{JL} \mathrm{Barlow}^{3}$, P Penumetcha ${ }^{1}$, M Pelletier ${ }^{1}$, G Sciumè ${ }^{4}$, \\ AC Richard ${ }^{1}$, ET Hayes ${ }^{1}$, J Gomez-Rodriguez ${ }^{5}$, X Chen ${ }^{6}$, WE Paul ${ }^{6}$, TA Wynn ${ }^{2}$, \\ ANJ McKenzie ${ }^{3}$ and RM Siegel ${ }^{1}$
}

The tumor necrosis factor (TNF)-family cytokine TL1A (TNFSF15) costimulates T cells and promotes diverse T cell-dependent models of autoimmune disease through its receptor DR3. TL1A polymorphisms also confer susceptibility to inflammatory bowel disease. Here, we find that allergic pathology driven by constitutive TL1A expression depends on interleukin-13 (IL-13), but not on T, NKT, mast cells, or commensal intestinal flora. Group 2 innate lymphoid cells (ILC2) express surface DR3 and produce IL-13 and other type 2 cytokines in response to TL1A. DR3 is required for ILC2 expansion and function in the setting of Tcell-dependent and -independent models of allergic disease. By contrast, DR3-deficient ILC2 can still differentiate, expand, and produce IL-13 when stimulated by IL-25 or IL-33, and mediate expulsion of intestinal helminths. These data identify costimulation of ILC2 as a novel function of TL1A important for allergic lung disease, and suggest that TL1A may be a therapeutic target in these settings.

\section{INTRODUCTION}

The tumor necrosis factor (TNF) superfamily of cytokines and receptors functions to regulate specific aspects of both innate and adaptive immunity. TL1A (TNFSF15), together with a number of other TNF-family cytokines including LIGHT, OX40L, CD30L, 4-1BBL, and TNF, functions to costimulate T lymphocytes. ${ }^{1}$ TL1A signals via DR3 (TNFRSF25), which is constitutively expressed on $\mathrm{T}$ cells and upregulated upon T-cell activation. ${ }^{2}$ TL1 A expression is tightly controlled, and is normally undetectable unless induced in myeloid and endothelial cells by pro-inflammatory stimuli signaling through Toll-like receptors and Fc receptors. ${ }^{2,3}$ TL1A can also be induced in $\mathrm{T}$ cells by stimulation through the T-cell receptor (TCR), enabling the possibility of autocrine TL1ADR3 signaling in T cells. ${ }^{4}$ TL1A, acting through DR3, activates $\mathrm{NF}-\kappa \mathrm{B}$ and MAP kinase signaling pathways and enhances proliferation and cytokine production in activated $\mathrm{CD} 4{ }^{+} \mathrm{T}$ lymphocytes, particularly when other costimulatory signals such as those delivered through $\mathrm{CD} 28$ are not present. ${ }^{5}$ Perhaps because of the restricted nature of TL1A expression, compared with other TNF family members, the effects of TL1A on T cells in vivo are mainly apparent at the site of tissue inflammation. DR3-deficient T cells expand normally during primary immune responses, but are defective in expansion and cytokine production in response to antigens presented in the context of inflamed tissue. TL1A-DR3 interactions are essential for the development of disease in T cell-dependent animal models of multiple sclerosis, rheumatoid arthritis, inflammatory bowel disease, and allergic lung disease. ${ }^{4,6-8}$ A role for TL1A in host defense against infection has thus far been limited to control T-cell responses to Salmonella and selected viral infections. ${ }^{9,10}$ These observations, coupled with linkage of polymorphisms in the TNFSF15 locus encoding TL1A to inflammatory bowel disease and detection of elevated levels of TL1A in affected tissue from rheumatoid arthritis and inflammatory bowel disease patients, ${ }^{11-14}$ have suggested that

${ }^{1}$ Immunoregulation Section, Autoimmunity Branch, NIAMS, NIH, Bethesda, Maryland, USA. ${ }^{2}$ Immunopathogenesis Section, Laboratory of Parasitic Diseases, NIAID, NIH, Bethesda, Maryland, USA. ${ }^{3}$ MRC Laboratory of Molecular Biology, Cambridge, UK. ${ }^{4}$ Lymphocyte Cell Biology Section, NIAMS, Bethesda, Maryland, USA. ${ }^{5}$ Genetic Disease Research Branch, NHGRI, NIH, Bethesda, Maryland, USA and ${ }^{6}$ Laboratory of Immunology, NIAID, NIH, Bethesda, Maryland, USA. Correspondence: RM Siegel, (rsiegel@nih.gov)

Received 2 August 2013; revised 14 November 2013; accepted 25 November 2013; published online 25 December 2013. doi:10.1038/mi.2013.114 
TL1A may be a pathogenic cytokine in a number of autoimmune diseases.

Another line of evidence suggesting a specific role for TL1ADR3 interactions in promoting allergic type 2 inflammation has emerged from studies of mice expressing TL1A constitutively. Transgenic mice expressing TL1A on either T cells or dendritic cells spontaneously develop small intestinal pathology characterized by muscular layer and goblet cell hyperplasia, mast cell infiltration, and increased mucous production. In mice expressing higher levels of TL1A, an immune cell infiltrate enriched in $\mathrm{CD} 4{ }^{+} \mathrm{T}$ cells also appears. ${ }^{15-17}$ Despite abundant levels of interleukin-13 (IL-13) and IL-5 expression, insufficient $\mathrm{T}$ helper (Th) $2 \mathrm{~T}$ cells were found in the intestine to explain the elevation of these cytokines; in fact a greater fraction of T cells in the lamina propria or mesenteric lymph node expressed IL-17 than IL-13 or IL-4. ${ }^{15-16}$ In addition, allergic pathology was preserved in TL1A transgenic mice crossed to an OT-II TCR transgenic Recombination Activating Gene-deficient background, which have a monoclonal naïve $\mathrm{T}$-cell repertoire. These data raised the possibility that cell types other than $\mathrm{T}$ cells may respond to TL1A to produce type 2 cytokines and promote allergic pathology in TL1A transgenic mice. Recent studies with DR3-deficient mice have also suggested roles for DR3 beyond $\mathrm{T}$-cell costimulation, implicating DR3 in diverse processes such as macrophage and osteoclast differentiation and corticostriatal innervation in the brain., ${ }^{78,19}$

Recently, distinct populations of lymphocytes lacking clonotypic antigen receptors, T, B, or NK cell surface markers were identified in tissues such as intestine, mesenteric fat, and lung. These cells, termed as innate lymphoid cells (ILCs), make up only a small proportion of tissue resident lymphocytes, but secrete large amounts of effector cytokines and have been shown to be essential components of a number of different immune pathologies and allergic responses. ${ }^{20,21}$ ILCs arise from a common lymphoid progenitor and require signaling through cytokines activating the common gamma chain and the transcription factors TCF-1, ROR $\alpha$, or ROR $\gamma$ t for their development. ${ }^{22-24}$ Innate lymphocytes can be divided into three broad groups based on their cytokine secretion patterns. Group 2 ILC (ILC2) secrete large amounts of IL-5 and IL-13 and can be critical for host defense against intestinal parasites and also contribute to allergic lung pathology together with other lymphocyte subtypes such as NKT cells. ${ }^{25-27}$

We hypothesized that in addition to its effects on $\mathrm{T}$ cells, TL1A may costimulate ILCs, particularly ILC2, accounting at least in part for the $\mathrm{T}$ cell-independent allergic intestinal pathology found in mice constitutively expressing TL1A. We found that ILC2 expressed surface DR3, and could be directly stimulated by TL1A to produce IL-13 and other type 2 immune cytokines. DR3 was required for the expansion of ILC2 in two models of allergic lung disease. However, ILC2 expansion and host defense against the parasite Nippostrongylus brasiliensis, which depends on IL-25 and IL-33, was intact in DR3-deficient mice. These data establish a novel role for the TNF superfamily cytokine TL1A as an activator of ILCs.

\section{RESULTS}

TL1A-induced intestinal pathology is dependent on IL-13 but not on $\mathrm{T}$ cells, mast cells, or commensal microbiota

Constitutive expression of TL1A either in T cells or in dendritic cells results in hyperplasia and inflammatory changes in the small intestine including macroscopic lengthening, thickening of the muscularis layer with mast cell infiltration, and goblet cell hyperplasia. These pathological changes were associated with marked induction of IL-13 and IL-5 in the small intestine and mesenteric lymph nodes, and elevated levels of circulating IL$13{ }^{15,16}$ To confirm that IL-13 signaling is required for the immunopathology induced by TL1A, we crossed TL1A transgenic mice to mice deficient in the $\alpha 1$ chain of the IL13 receptor, which is required for IL-13 signaling in both immune and non-immune cells. The intestines of TL1A transgenic mice lacking IL-13R $\alpha 1$ were grossly normal and had normal mucosal thickness and goblet cell size (Figure 1a) showing that chronic TL1A expression induces pathology through the IL-13 signaling pathway.

To determine the relative contribution of T cells to TL1Ainduced intestinal pathology, we used CD11c-TL1A transgenic mice expressing TL1A in dendritic cells, which develop small intestinal pathology similar to CD2-TL1A transgenic mice. ${ }^{15,16}$ These mice were crossed onto a TCR $\alpha$-deficient background. Surprisingly, small intestinal pathology developed in TL1A transgenic mice despite the lack of $\alpha \beta$ T cells in these mice, with comparable increases in mucosal thickness and goblet cell area in the ileum with or without $\alpha \beta T$ cells (Figure 1b). IL-13 expression in TCR $\alpha$-deficient TL1A transgenic mice was still significantly elevated (Figure 1c). Although $\gamma \delta$ TCR expressing $\mathrm{T}$ cells still develop in TCR $\alpha$-deficient mice, goblet cell hyperplasia and IL-13 production in the small intestine are also preserved in TL1A transgenic mice on a Recombination Activating Gene-1-deficient background which prevents $\gamma \delta$ T-cell development. ${ }^{16}$ Thus, it is unlikely that either $\alpha \beta$ or $\gamma \delta \mathrm{T}$ cells are required for IL-13 production and intestinal pathology in response to TL1A.

Other cell types besides T cells may be the source of IL-13 induced by constitutive TL1A. Invariant NKT cells producing IL-13 have been implicated in some mouse models of colitis, but are relatively depleted in TL1A transgenic mice. ${ }^{16}$ Complete deletion of iNKT cells by backcrossing to J $\alpha 18$-deficient mice or all NKT cells in CD1d-deficient mice did not ameliorate small intestinal pathology or IL-13 production in TL1A transgenic mice (Supplementary Figure S1 online). Mast cells accumulate in the submucosa of TL1A transgenic mice and can also produce IL-13 and IL-5, but depletion of mast cells on the W/ $\mathrm{W}^{\mathrm{v}}$ background was ineffective at reducing small intestinal hyperplastic changes in TL1A transgenic mice and only marginally effective in reducing IL-13 production (Supplementary Figure S1). Taken together, these data suggest that neither NKT nor mast cells are the major sources of IL-13 responsible for intestinal pathology in TL1A transgenic mice.

Intestinal microbiota can profoundly influence the development and cytokine secretion potential of $\mathrm{T}$ cells and innate 
a

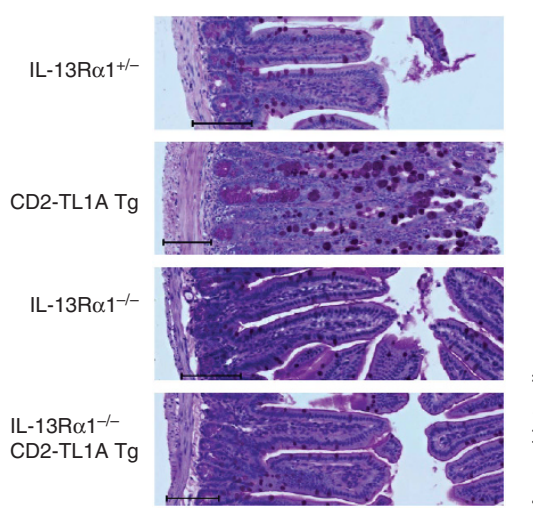

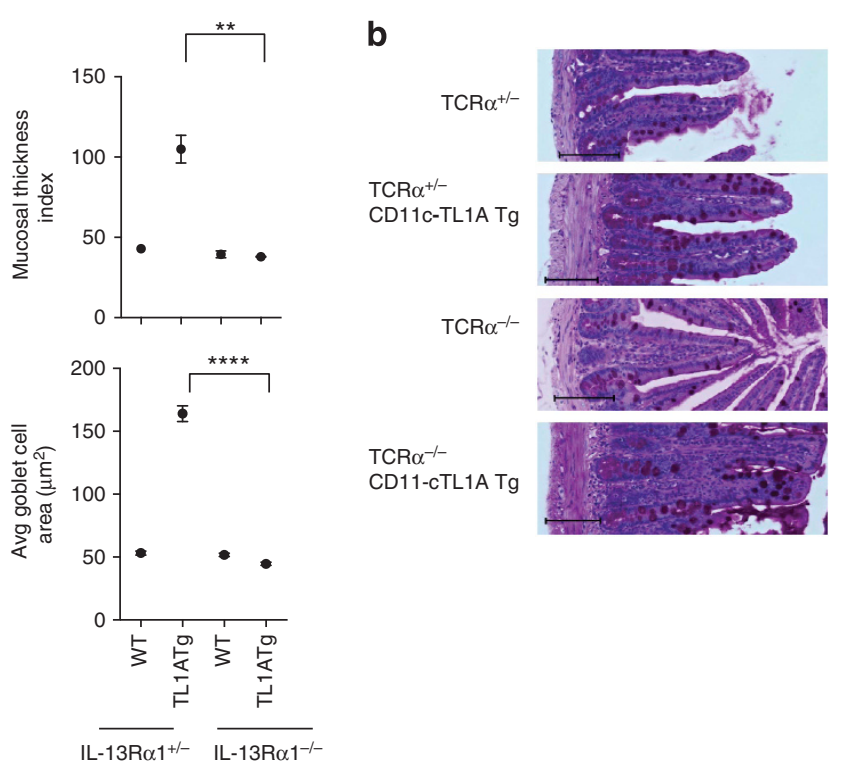
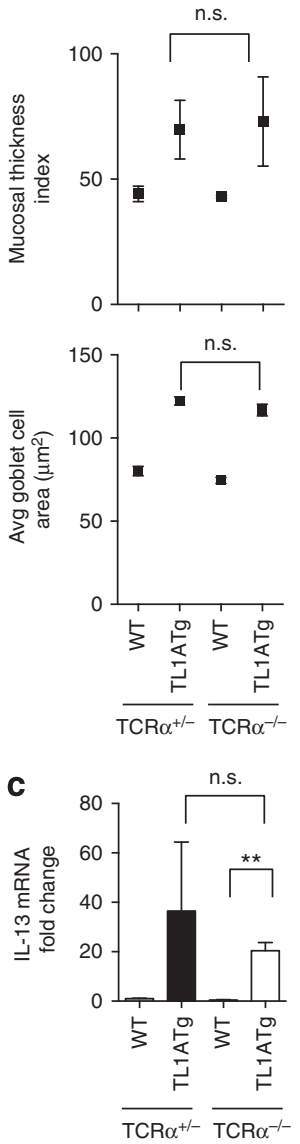

Figure 1 Small bowel pathology in TL1A transgenic (Tg) mice is interleukin-13 (IL-13) dependent but does not require $\alpha \beta$ T cells. (a) CD2-TL1A Tg mice were crossed to IL13R $\alpha 1$-deficient mice. Representative transverse histological ileum sections stained with Periodic Acid Schiff (PAS) for goblet cells from 20-week-old mice of the indicated genotypes are shown. Scale bar $=100 \mu \mathrm{m}$. Mucosal thickness index and goblet cell area for three mice per group are shown with each dot representing the average within a group (mean \pm s.e.m.) with significance from the Mann-Whitney test $\left.{ }^{* *} P<0.01,{ }^{* * \star \star} P<0.0001\right)$. (b) CD11c-TL1A Tg mice were crossed to TCR $\alpha^{-1-}$ mice. Representative transverse histological ileum sections stained with PAS from 15-week-old mice from the indicated genotypes are shown. Scale bars $=100 \mu \mathrm{m}$. Mucosal thickness and goblet cell area from three mice per group are shown at right with each dot representing the average within a group. (c) IL-13 mRNA from ileal tissue was measured by qRT-PCR, normalized to $\beta 2 \mathrm{~m}$ expression and shown relative to expression levels in wild-type (WT) mice. Data shown are the mean \pm s.e.m. analyzed, with significance shown from the Mann-Whitney test. See also Supplementary Figure S1. TCR, T-cell receptor.

immune cells in the gut. ${ }^{28}$ We previously found that restricting the T-cell repertoire with the OT-II TCR transgene did not reduce the intestinal hyperplasia in TL1A transgenic mice, ${ }^{16}$ suggesting that $\mathrm{T}$-cell antigens derived from intestinal microbiota were not required. To determine whether innate immune IL-13 producing cells induced by TL1A require the presence of commensal intestinal flora, we treated CD2-TL1A transgenic mice with oral antibiotics from pregnancy or from birth until 3 months, an age at which significant intestinal pathology has developed in most TL1A transgenic mice. Antibiotic treatment did not prevent the development of goblet cell and intestinal hyperplasia in the presence of TL1A; in fact both parameters were significantly increased in antibiotic-treated compared with age-matched control TL1A transgenic mice (Figure 2a). TL1A transgenic mice depleted of commensal flora also had even more elevated levels of IL-13 mRNA (Figure 2b). Microbial depletion was adequate in these mice, as assessed by reduction in stool DNA concentration (Figure 2c). The effects of microbial depletion could also be seen through the reduction in percentages of memory $\mathrm{T}$ cells in antibiotic-treated mice, particularly in the mesenteric lymph nodes (Figure 2d). These results strongly suggest that chronically expressed TL1A drives IL-13 production and intestinal pathology in the absence of immune stimuli derived from commensal microbial flora.

\section{TL1A stimulates ILC2 to produce IL-13 and other type 2 immune cytokines}

ILC2 lack T, B, or NK cell lineage markers, and express the transcription factor GATA-3 and the surface proteins Thy-1, ICOS, and KLRG1 ${ }^{21,29}$ ILC2 produce large quantities of IL-13 and IL-5 (but little or no IL-4) in response to the cytokines IL-25 or IL-33. ILC2 expansion and cytokine secretion can also be triggered in the setting of gastrointestinal parasitic infection and allergic lung disease. ${ }^{21,25-27,30-32}$ However, whether these cells express TNF-family receptors or receive costimulatory signals through these receptors remains not known. To determine whether TL1A might act directly on ILC through its receptor DR3, we measured surface expression of DR3 on 
a
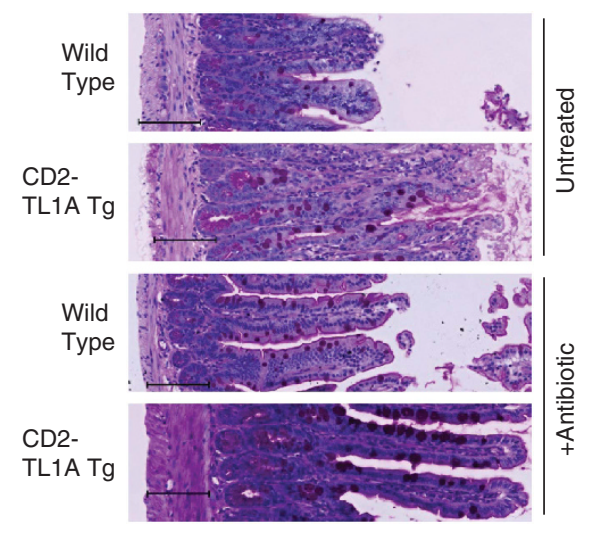
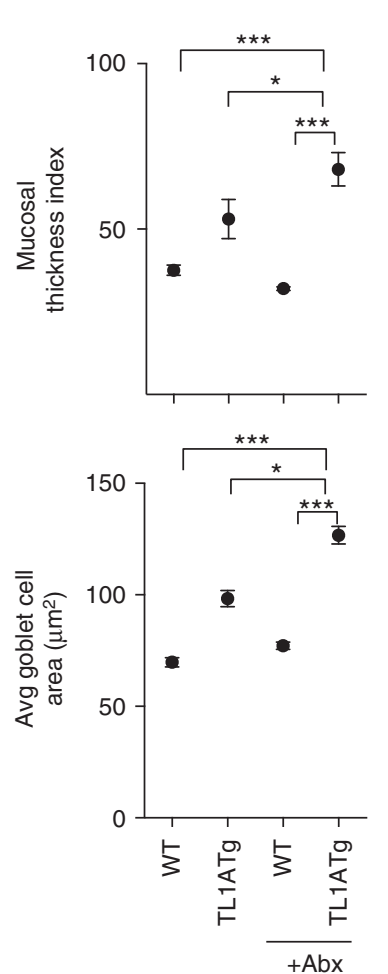

b
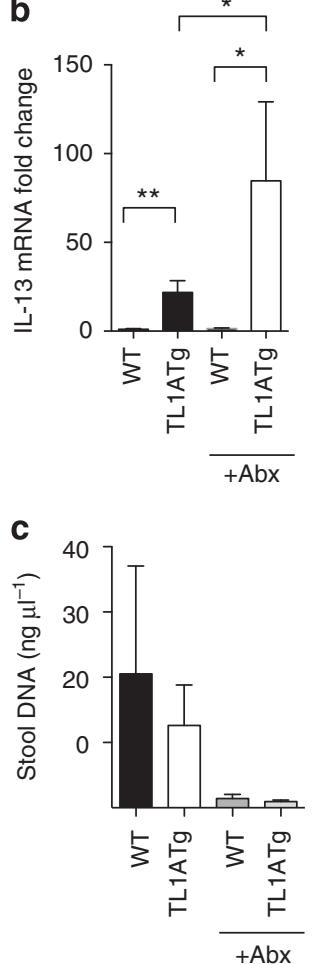

d

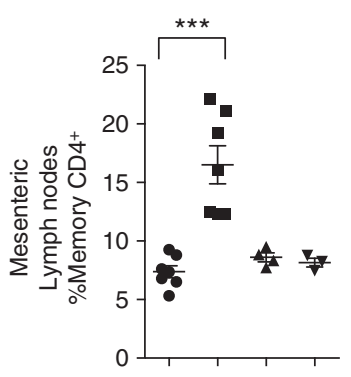

Figure 2 Commensal bacteria are not required for TL1A-driven small intestinal pathology. (a) CD2-TL1A transgenic (Tg) mice were treated with a quadruple antibiotic regimen for 3 months. Representative transverse histological ileum sections stained with Periodic Acid Schiff (PAS) from 12-week-old mice. Scale bar $=100 \mu \mathrm{m}$. Mucosal thickness and goblet cell area are shown at right for 3-6 mice per group with each dot representing the average within a group (mean \pm s.e.m.) with significance from the Mann-Whitney test $\left({ }^{\star} P<0.05,{ }^{\star \star \star} P<0.001\right)$. (b) Interleukin-13 (IL-13) mRNA from ileal tissue was quantitated by qRT-PCR, normalized to $\beta 2 \mathrm{~m}$ expression and shown relative to expression levels in untreated WT mice. Data represent mean \pm s.e.m with significance from the unpaired $t$-test $\left({ }^{\star} P<0.05,{ }^{* *} P<0.01,{ }^{* * *} P<0.001\right)$. (c) Total DNA isolated from mouse fecal matter. (d) The percentage of memory $\mathrm{CD} 4{ }^{+}$cells $\left(\mathrm{CD} 44^{\mathrm{hi}} \mathrm{CD} 62 \mathrm{~L}^{\mathrm{lo}}\right)$ in the spleen and mesenteric lymph nodes measured by flow cytometry. Data shown are the mean \pm s.e.m. for 3-6 mice per group.

lymphocytes from mesenteric lymph nodes and found that Lin $^{-}$Thyl $^{+}$cells constitutively express DR3 at high levels, comparable to that on $\mathrm{CD} 4{ }^{+} \mathrm{T}$ cells (Figure $3 \mathrm{a}$ ). More detailed analysis of lineage-negative lymphocytes using markers more specific for different groups of ILC $^{21,29}$ revealed that ILC2 (NK1.1 ${ }^{-}, \mathrm{KLRG}^{+}{ }^{+}$, or GATA3 $\left.{ }^{+}\right)$and ILC3 $\left(\mathrm{ROR} \gamma \mathrm{t}^{+}\right.$, $\mathrm{CD}_{127^{+}}$) expressed higher levels of DR3 than ILC1 (NK1.1 ${ }^{+}$ $\mathrm{KLRG}^{+}$) (Supplementary Figures S2a and b). All three of these ILC subsets appeared to be present in normal numbers in DR3-deficient mice (Supplementary Figure S2c), indicating that DR3 was not required for differentiation or homeostasis of ILC.

To determine whether TL1A could directly induce IL-13 production by ILC2, we isolated lineage (lin) ${ }^{-}$ICOS $^{+}$ILC2 from the mesenteric lymph nodes and spleen of $I l 13^{e G F P /+}$ reporter mice treated with IL-25 to expand numbers of these cells. TL1A induced expression of the IL13-GFP reporter in a dose-dependent manner, which was enhanced by the addition of IL-7, which is known to promote ILC2 survival and cytokine secretion (Figure $\mathbf{3 b}, \mathbf{c}$ ). Analysis of supernatants from stimulated ILC2 showed that TL1A was effective in inducing an ILC2 "cytokine signature" in vitro, including IL-5, IL-6, and IL-13, with much smaller quantities of IL-9 or IL-17A (Figure 3d). Addition of IL-7 enhanced secretion of these cytokines. These data show that TL1A can directly stimulate ILC2 to enhance secretion of type 2 cytokines.

In addition to enhancing the function of ILC2, IL-25 and IL33 injected in vivo can induce rapid expansion of ILC2 (Figure 4a). However, injection of up to $5 \mu \mathrm{g}$ of recombinant TL1A for three consecutive days failed to expand ILC2 or induce expression of the IL-13eGFP reporter in $\mathrm{Lin}^{-} \mathrm{ICOS}^{+}$ cells (Figure 4a). In CD2-TL1A transgenic mice, chronic TL1A expression did not expand ILC2 in the mesenteric lymph nodes and small intestinal lamina propria as occurs with IL-25 treatment (Figure $4 \mathrm{~b}$ ). However, small numbers of IL-13eGFP reporter expressing cells were found in TL1A transgenic mice, with cells in the mesenteric lymph node expressing high levels of the eGFP reporter (Figure $\mathbf{4 b}$ ). To test the effects of TL1A in an alternate reporter system in which both endogenous alleles of IL-13 remain intact, we used BAC transgenic reporter mice with a DsRED reporter replacing the IL-13 coding region and an AmCyan reporter replacing the IL-4 coding region (4C13R mice). ${ }^{33}$ The TL1A transgene induced small numbers of $\mathrm{Lin}^{-} \mathrm{ICOS}^{+}$cells in the mesenteric lymph nodes of 4C13R mice to produce the IL-13 reporter (Supplementary Figure S3a), comparable to the suboptimal dose of IL-25 used in these experiments. Again, as in IL-13eGFP reporter mice, there was no expansion of the $\mathrm{Lin}^{-} \mathrm{ICOS}^{+}$population in TL1A 
a<smiles>[10BH2]</smiles>

Isotype
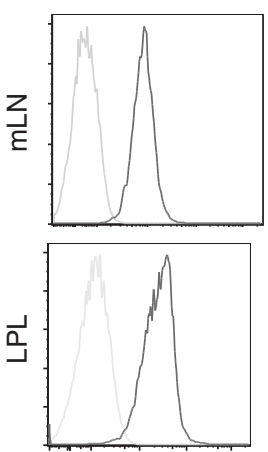

Lin-Thy ${ }^{+}$
- DR3

Isotype
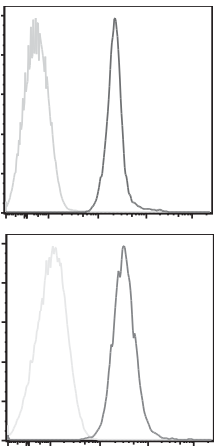

$\mathrm{CD} 4^{+} \mathrm{T}$ cell
- $\mathrm{CD}^{+}$

- Lin-Thy $1^{+}$
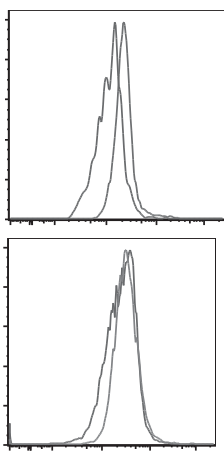

C

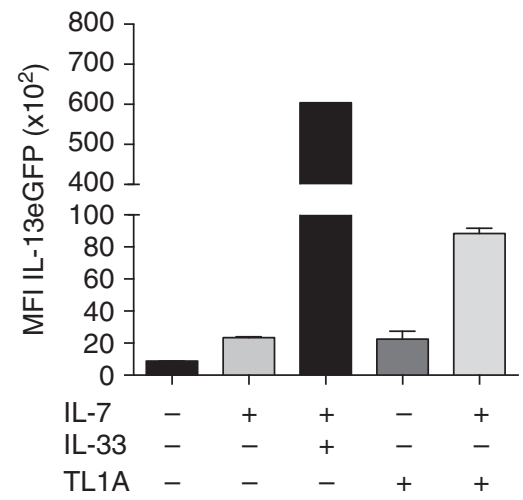

b
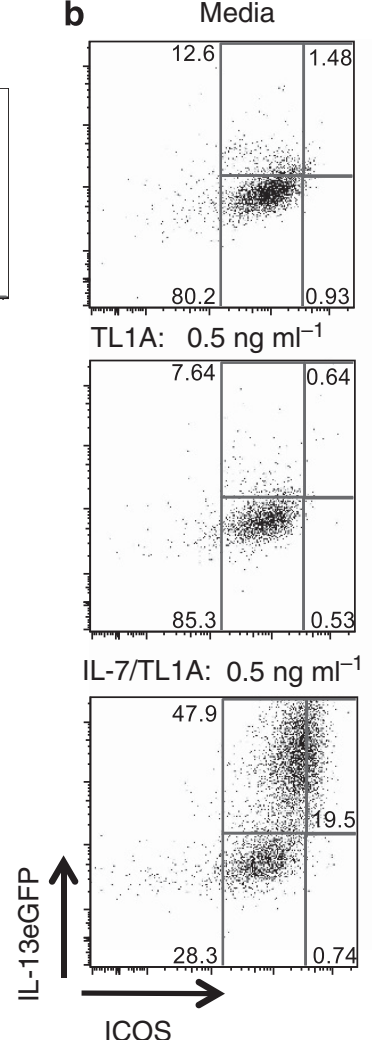

IL-7

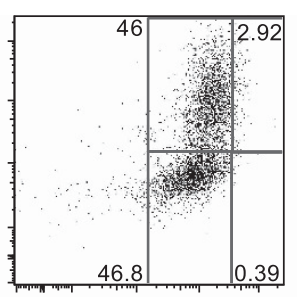

$5 \mathrm{ng} \mathrm{m}^{-1}$
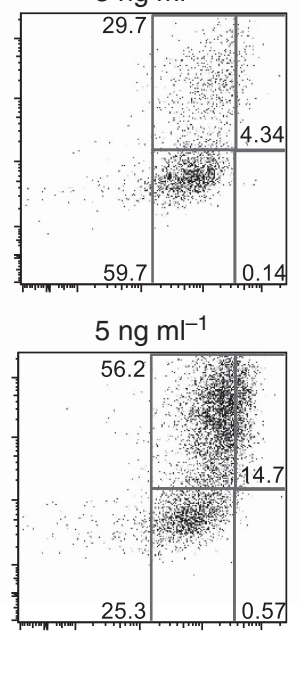

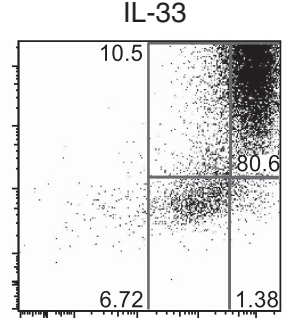

$50 \mathrm{ng} \mathrm{ml}^{-1}$
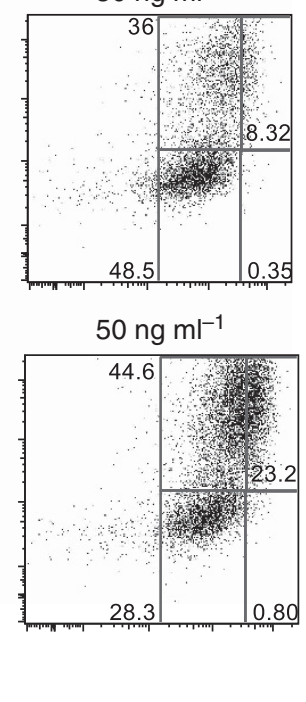
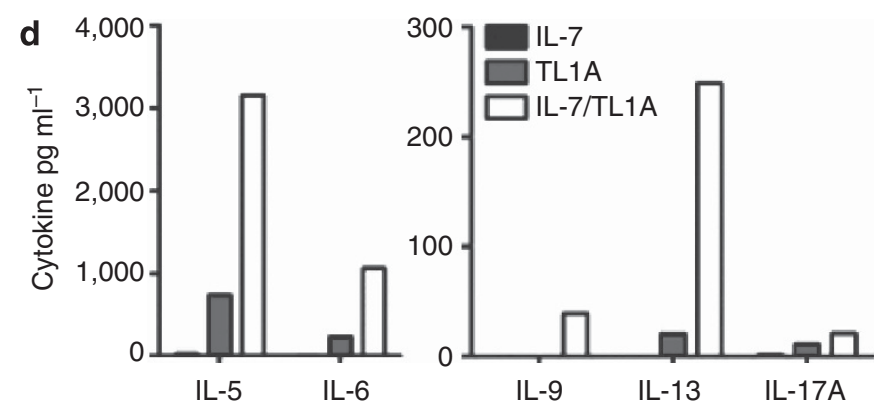

Figure 3 TL1A is a costimulatory cytokine for group 2 innate lymphoid cells (ILC2). (a) Surface expression of DR3 was measured by flow cytometry in $\mathrm{Lin}^{-}$Thy $1^{+}$lymphocytes vs. CD4 ${ }^{+} \mathrm{T}$ cells in mesenteric lymph nodes $(\mathrm{mLN})$ and the intestinal lamina propria. (b) Lineage-negative/ ICOS-positive cells were purified from interleukin-25 (IL-25)-treated $/ 113+$ leGFP mice and treated with the indicated cytokines (IL-7 at $10 \mathrm{ng}$ ml ${ }^{-1}$, $\mathrm{IL}-33$ at $10 \mathrm{ng} \mathrm{ml}^{-1}$ and the indicated concentration of TL1A) for $72 \mathrm{~h}$ and then assayed for expression of the IL-13eGFP reporter by flow cytometry. Dot plots are gated on lineage-negative cells. (c) Mean fluorescence intensity (MFI) from data presented in (b). (d) Lineage (CD3CD4CD8CD19CD11bCD11cFceR1Gr1)-negative ICOS-positive ILCs were purified from IL-25-treated I/13+/eGFP mice, and treated with $10 \mathrm{ng} \mathrm{ml}^{-1}$ of the indicated cytokines for $72 \mathrm{~h}$ before collection of supernatants for cytokine measurements. See also Supplementary Figure S2. LPL, lamina propria lymphocyte.

transgenic mice. Induction of cells expressing the IL-4 reporter was much weaker than IL-13, consistent with the poor ability of ILC2 to produce IL-4 (Supplementary Figure S3b). To determine whether chronically expressed TL1A may induce IL-25 and/or IL-33, which may serve to sustain IL-13 production by ILC2, we measured expression of these two cytokines in the small intestine, and found that indeed expression of IL-25, and to a lesser extent, IL-33, was elevated in CD2-TL1A transgenic mice (Figure 4c). Taken together, these data show that transgene-driven expression of TL1A can induce intestinal ILC2 to spontaneously produce
IL-13 without causing the massive expansion of this subset that can be seen with extrinsic administration of IL-25 or IL-33.

\section{Expansion of ILC2 in allergic lung disease but not in parasitic infection depends on TL1A-DR3 interactions}

A number of features of the intestinal pathology in TL1A transgenic mice resemble those that occur in the small intestine during responses to intestinal parasites, such as $N$. brasiliensis. IL-25, IL-33, and ILC2-derived IL-13 are known to play key roles in immunity to $\mathrm{N}$. brasiliensis. ${ }^{34,35}$ These findings raised the possibility that TL1A may promote host defense against 


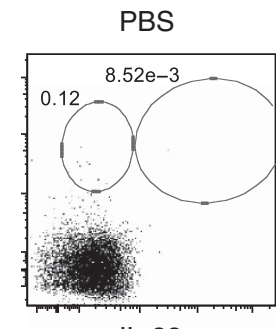

IL-33

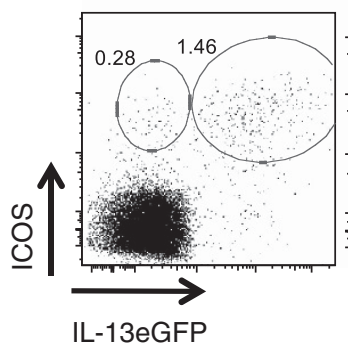

b

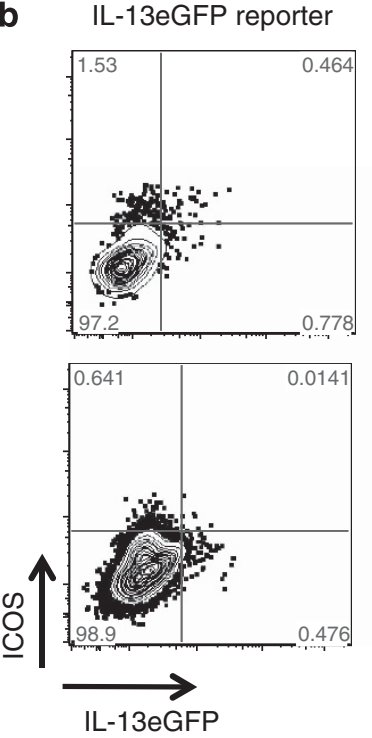

IL-13eGFP+ IL-25
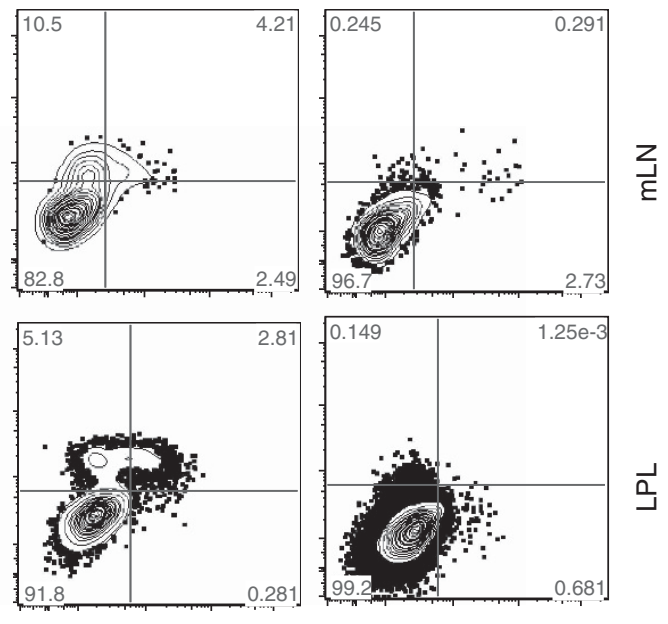

IL-13eGFPx CD2-TL1ATg

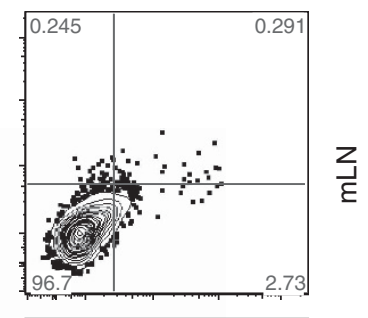

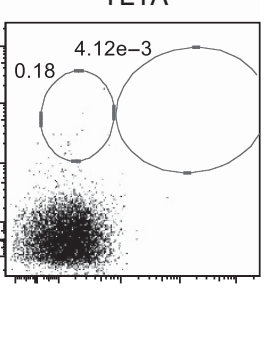

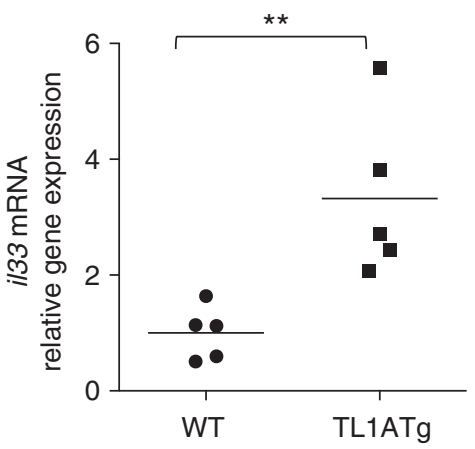

Figure 4 Chronic, but not acute exposure to TL1A can induce interleukin-13 (IL-13) expression in innate lymphoid cell (ILC). (a) ICOS and IL-13eGFP expressing cells were quantitated in mesenteric lymph node $(\mathrm{mLN})$ following four consecutive doses over 4 days of phosphate-buffered saline (PBS),

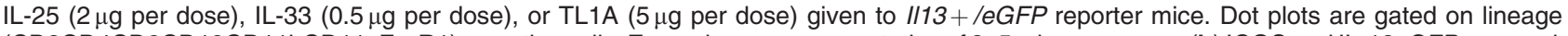
(CD3CD4CD8CD19CD11bCD11cFceR1)-negative cells. Examples are representative of 3-5 mice per group. (b) ICOS and IL-13eGFP expressing cells were quantitated in $\mathrm{mLN}$ and lamina propria from $/ 113+$ /eGFP reporter mice untreated, given $2 \mu \mathrm{g}$ of IL-25 for four consecutive days, or crossed to CD2TL1A transgenic (Tg) mice. (c) il25 and il33 mRNAs were measured by qRT-PCR in the ileum of CD2-TL1A Tg mice and normalized to the average levels found in wild-type (WT) age-matched controls and analyzed with Mann-Whitney test $\left({ }^{\star \star} P<0.01\right)$. See also Supplementary Figure S3.

N. brasiliensis by stimulating ILC2 to produce IL-13. To test this hypothesis, we infected wild-type and DR3-deficient mice with $N$. brasiliensis and measured the ability of DR3-deficient mice to mount an anti-parasitic immune response, and clear the parasite efficiently from the intestines, as most wild-type mice do by 14 days post infection. Contrary to this notion, DR3deficient mice mounted a robust response to infection, with significantly more goblet cell hyperplasia and mucosal thickening in the duodenum, the most affected portion of the small intestine, than wild-type controls (Figure 5a,b). Numbers of adult worms in the small intestine of DR3-deficient mice were lower at the peak of infection both at day 6 and at day 14 compared with wild-type mice, with corresponding reductions in the number of eggs in the stool (Figure 5c), indicating highly effective immunity to $N$. brasiliensis in the absence of DR3.

Induction of IL-13 expression in the lung, which the larvae pass through before reaching the intestine, was similar in wildtype and DR3-deficient mice, indicating that the reduced parasitemia in the intestine is not simply the result of lower overall infection efficiency in DR3-deficient mice. IL-13 induction was also robust in the duodenum of DR3-deficient mice exposed to $N$. brasiliensis, with more sustained elevation in IL-13 mRNA (Figure 5d). Furthermore, systemic levels of IL-13 protein and its secreted receptor, IL-13R $\alpha 2$, a marker of IL-13 bioactivity, also increased in infected DR3-deficient mice with similar kinetics and with equal saturation of IL-13R $\alpha 2$ by IL-13 (Figure 5e). ILC2 also expanded robustly in DR3deficient mice infected with $N$. brasiliensis (Figure 5f), suggesting that helminth-driven expansion of ILC2 is independent of DR3. IL-33 and IL-25 are necessary to promote expansion of ILC2 during Nippostrongylus infection and sufficient to stimulate ILC2 expansion on their own. ${ }^{34,35}$ To test whether DR3 is required for expansion of ILC2 by these cytokines, we treated DR3-deficient and control wild-type mice with IL-25 and IL-33. ILC2 expanded normally in response to either IL-25 or IL-33 in DR3-deficient mice (Supplementary Figure S4) showing that ILC expansion 
a

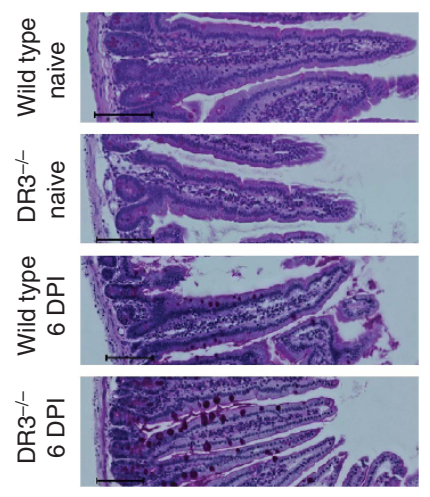

c

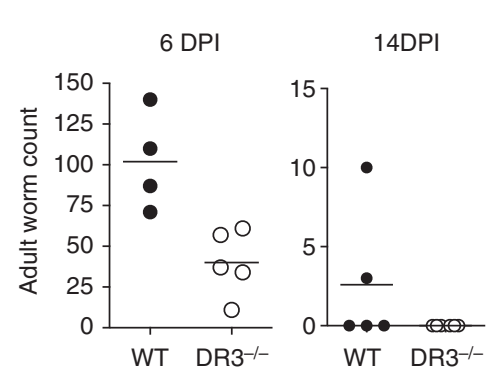

b
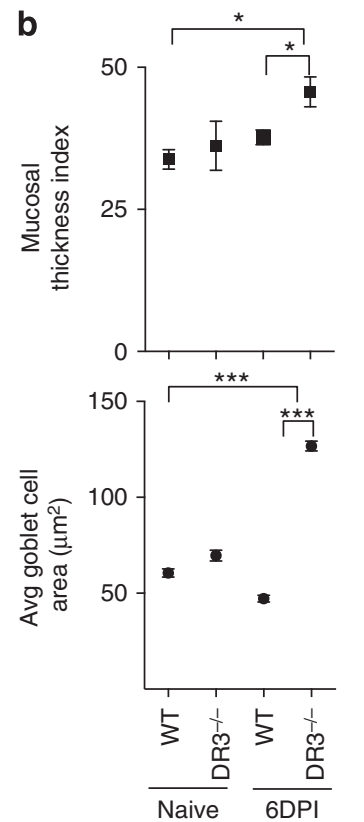
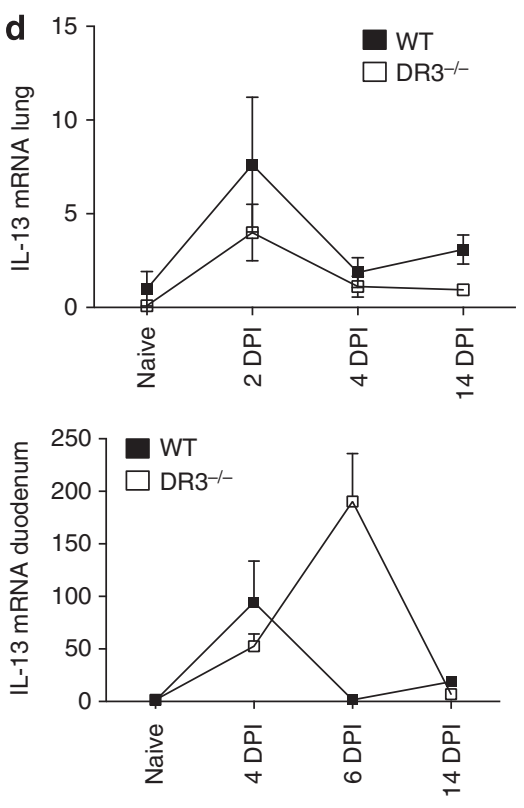

e

Total IL-13R $\alpha 2$ IL-13/IL-13R $\alpha 2$

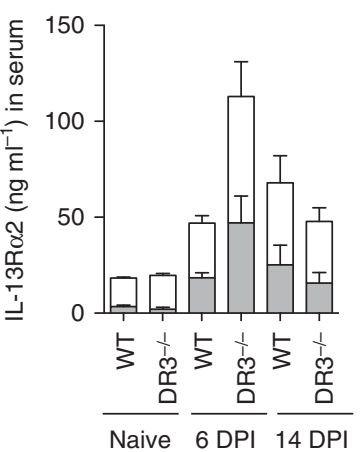

f

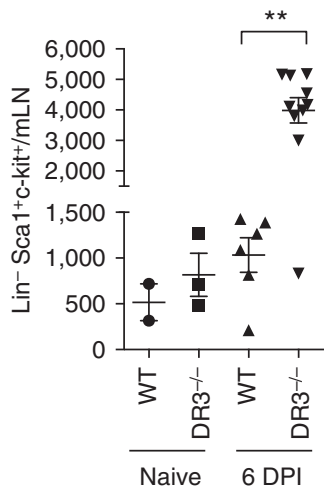

Figure 5 DR3 is not required for interleukin-13 (IL-13) production and clearance of parasites in Nippostrongylus brasiliensis infection. (a) Representative Periodic Acid Schiff (PAS)-stained duodenum sections from wild-type (WT) or DR3 ${ }^{-1-}$ mice without or 6 days post infection (DPI) with N. brasiliensis. Scale bar $=100 \mu \mathrm{m}$. (b) Mucosal thickness and goblet cell area in these samples are shown for 4-9 mice per group with each dot representing the average within a group. Data represent mean \pm s.e.m., analyzed with Mann-Whitney test $\left({ }^{*} P<0.05\right.$, $\left.{ }^{\star * \star} P<0.001\right)$. (c) Adult worm counts from the intestine at 6 and $14 \mathrm{DPI}$, and counts of eggs in the stool over the course of infection. Data represent mean \pm s.e.m. (d) IL-13 mRNA expression in the duodenum over the course of infection normalized to $\beta 2 \mathrm{~m}$ expression and shown relative to expression levels in untreated WT mice with mean \pm s.e.m. (e) Serum levels of IL-13R $\alpha 2$ (white bars) and IL-13 bound to IL-13R $\alpha 2$ (gray bars) measured in serum at the indicated times after N.brasiliensis infection in the indicated mice. (f) Group 2 innate lymphoid cells (ILC2) quantitated in mesenteric lymph nodes of uninfected and 6 DPI Nippostrongylus-infected mice using Lin ${ }^{-}$(CD3CD4CD8CD11bCD19MHCIIFcERI) ckit ${ }^{+}$Sca1 $^{+}$as markers of ILC2. Significance was tested by Mann-Whitney test $\left({ }^{\star \star} P<0.01\right)$. See also Supplementary Figures S4 and S5.

in this setting is largely independent of DR3. Taken together, these data show that despite the similarity in pathology between TL1A transgenic mice and helminth-infected mice, DR3 is not required to expand ILC2 or mount a protective immune response to $\mathrm{N}$. brasiliensis.

Other parasitic infections may require DR3 to induce a type 2 immune response. To test this, we infected wild-type and DR3deficient mice with Schistosoma mansoni. During schistosomal infection, host IL-13 drives the granulomatous response that contains parasite eggs in the liver, but also promotes hepatic fibrosis. ${ }^{36,37}$ In response to $S$. mansoni infection, DR3-deficient mice produced at least as much IL-13, IL-10, and interferon- $\gamma$ as wild-type C57BL/6 control mice (Supplementary Figures S5a and $\mathbf{b}$ ). The hepatic response to S. mansoni also appeared to be normal in DR3-deficient mice, with comparable size and percentage of eosinophils in liver granulomas, and liver weight, a measure of hepatomegaly (Supplementary Figure S5c). DR3-deficient mice and controls also had comparable worm and egg counts (Supplementary Figure S5d). The only defect seen in DR3-deficient mice was lower leukocyte cell yields, consistent with the known ability of DR3 to costimulate T-cell expansion (Supplementary Figure S5e). Hepatic fibrosis was similarly proportional to the burden of infection in wild-type and DR3-deficient mice (Supplementary Figure S5f). These data show that the immune responses to the helminth parasites $S$. mansoni and $N$. brasiliensis develop independently of costimulation through DR3. 

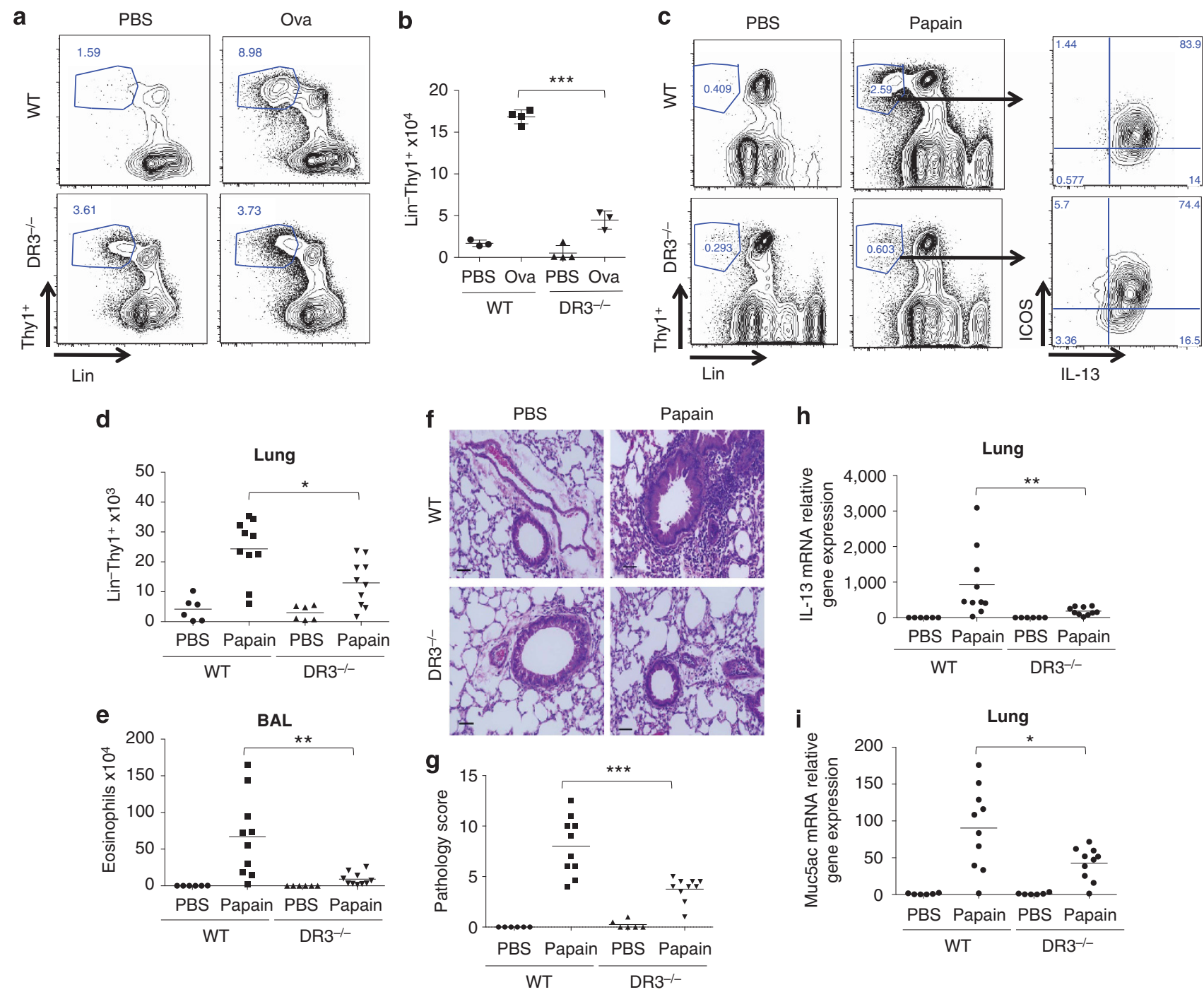

Figure 6 DR3 is required for group 2 innate lymphoid cells (ILC2) expansion and lung pathology during allergic lung disease. (a) Percentage of Lin (CD3,CD4,CD8,CD11b,CD11c,B220,GR1,Ter119,NK1.1) Thy1 ${ }^{+}$ILC gated in CD45 ${ }^{+}$population in the lungs of mice of the indicated genotype immunized and challenged with ovalbumin or phosphate-buffered saline (PBS) control. (b) Quantitation of Lin ${ }^{-}$Thy $1^{+}$cells from the lungs of mice immunized and challenged as in (a). Each point represents one mouse with mean \pm s.e.m., with significance from the unpaired $t$-test $\left.{ }^{* * \star} P<0.001\right)$. (c) Percentage of Lin $^{-}$(CD3,CD4,CD8,CD11b,CD11c,B220,GR1,Ter119,NK1.1) Thy 1 ${ }^{+}$ILC gated in CD45 ${ }^{+}$population in the lungs of mice of the indicated genotype intranasally challenged with papain or control (PBS). (d) Quantitation of Lin ${ }^{-}$Thy $1^{+}$cells from the lungs of mice immunized and challenged as in (c). (e) Numbers of eosinophils in the bronchoalveolar lavage (BAL) of these mice, calculated from yield and flow-cytometric assessment of BAL as described in Methods. (f) Representative histological sections from the lungs of mice challenged with Papain as in (c). Hematoxylin and eosin $(\mathrm{H} \& \mathrm{E})$ staining, scale bar $=50 \mu \mathrm{m}$. Quantitation of immunopathology in lung histological sections examined from groups of mice treated as in (c) is shown in (g). Scoring was performed as described in Methods. Each symbol represents the score of one mouse. Expression of il13(h) and muc5ac (i) mRNA in lung tissue isolated from the mice treated as in (c) measured by qRT-PCR and normalized to the average levels found in wild-type (WT) age-matched controls. Data were analyzed with Mann-Whitney test $\left({ }^{\star} P<0.05\right.$, $\left.{ }^{\star \star} P<0.01,{ }^{\star \star \star} P<0.001\right)$.

To determine the physiological role of DR3 on ILC2 in the context of allergic disease, we measured ILC numbers in the lungs of mice induced to develop hypersensitivity pneumonitis to ovalbumin, a mouse model for allergic asthma. Using this model system, we have previously shown that DR3-deficient mice have reduced pathology and IL-13 production in the lung despite a normal systemic Th2 response to ovalbumin. ${ }^{4}$ However, the role of ILC in this defect has not been previously investigated. In contrast to the exaggerated expansion of ILC2 in the setting of Nippostrongylus infection, expansion of Lin ${ }^{-}$ Thy $1^{+}$lymphocytes was curtailed in the lungs of DR3- deficient mice, with $\sim 2.5$-fold fewer cells with this phenotype in the lungs of DR3-deficient mice challenged with ovalbumin compared with wild-type mice (Figure 6a,b).

To study the requirement for DR3 on ILC2 in an allergic response in which innate immune cells have a more central role, we exposed wild-type and DR3-deficient mice to inhaled papain, an environmental plant allergen whose cysteine protease activity triggers an acute allergic response through direct activation of innate immune cells including basophils and inflammatory dendritic cells. ${ }^{38,39}$ Papain-induced allergic lung pathology is only partially dependent on the presence of 
T cells with T-cell dependence not observed until at least 2 weeks of exposure to papain. ${ }^{31,40}$ In response to exposure to four doses of inhaled papain over 6 days, Lin ${ }^{-}$ILCs expanded poorly in DR3-deficient mice compared with wild-type C57Bl/6 controls (Figure 6c). Restimulation of isolated cells with phorbol 12myristate 13-acetate and ionomycin revealed that almost all $\mathrm{Lin}^{-}$Thy $1^{+}$in papain-treated mice were able to produce IL-13. However, significantly fewer Lin ${ }^{-}$Thy $1^{+}$cells were found in the lungs of papain-challenged DR3-deficient mice compared with wild-type controls (Figure 6d). These defects had significant consequences for airway inflammation, as DR3deficient mice challenged with papain had dramatically reduced airway eosinophila and lung pathology, including greatly diminished peribronchiolar infiltration and goblet cell hyperplasia (Figure $\mathbf{6 e}-\mathbf{g}$ ). Expression of IL-13 and Mucin $5 \mathrm{ac}$, which are associated with goblet cell metaplasia and increased mucous production, was also significantly reduced in the lungs of papain challenged DR3-deficient mice (Figure 6h,i). Taken together, these data show that TL1ADR3 interactions are important for the innate lymphocyte response and disease severity in two distinct models of allergic lung disease, the latter primarily dependent on ILC2.

\section{DISCUSSION}

While transcription factors such as TCF- 1 and ROR $\alpha$ have been identified as being important for the development of ILC2 from lymphoid precursors, ${ }^{23,24}$ the signals that promote ILC2 to secrete type 2 cytokines have not been as well defined. Here, we uncover costimulation of ILC2 as a new function for TL1A. TL1A induces expression of IL-13 and other type 2 cytokines in ILC2, likely contributing to the allergic intestinal phenotype seen in mice constitutively expressing TL1A. This effect is direct, in that TL1A can induce IL-13 expression in purified ILC. However, in vivo, unlike IL-25 or IL-33, TL1A does not expand ILC2 numbers or acutely induce IL-13 expression in these cells by itself. Over time, likely due to the presence of low levels of endogenous stimulators, chronic TL1A expression in TL1A transgenic mice induces IL-13 production by ILC2 without expanding them. The fact that TL1A transgenic mice treated with antibiotics developed more severe allergic intestinal pathology suggests that commensal gut flora do not provide this endogenous stimulation, and may actually provide inhibitory signals that constrain the effects of TL1A on ILC2. Low levels of IL-33 secreted during epithelial cell turnover in the intestine may be one possible source of a primary signal for ILC2 in this setting, as it was recently reported that IL-33 is constitutively expressed by epithelial cells from barrier tissues. ${ }^{41}$ We propose that TL1A should be thought of as a costimulator or "signal 2" which fully activates ILC function, analogous to its costimulatory role in T-cell biology. Whether or not other TNF-family cytokines that act on lymphocytes can also costimulate ILCs will be an important topic for future study.

These findings reveal a novel role for TL1A in the biology of ILCs, and prompt re-examination of findings in DR3-deficient mice to consider effects on ILC in addition to $\mathrm{T}$ cells. The defective expansion of ILC2 in the lung during ovalbumin and papain induced lung hypersensitivity suggests that the function of ILC2 during allergic lung disease also depends on TL1A-DR3 interactions. In this model, the defect in ILC2 expansion may be secondary to the defective expansion of DR3-deficient $\mathrm{T}$ cells after ova challenge in the lung. ${ }^{4}$ In acute papain-induced allergic lung disease, ILC2 can expand and produce IL-13 independently of $\mathrm{T}$ cells, ${ }^{24,42}$ suggesting that defective ILC2 expansion in response to papain in DR3-deficient mice is due to a cell-intrinsic requirement for TL1A costimulation by these cells.

Remarkably, although TL1A-DR3 interactions are required for the ILC2 cell number expansion and functional activation in sterile allergic responses, host defense against the parasites $N$. brasiliensis and $S$. mansoni is not compromised. This divergence is particularly interesting given that allergens and parasites share many common pathways for activating a type 2 immune response, including induction of the ILCexpanding cytokines IL-25 and IL-33. ${ }^{43}$ One possibility is that quantities of IL-25 and/or IL-33 induced by N. brasiliensis parasites are sufficient to bypass the need for costimulation of ILC2 through DR3. Alternatively, a cytokine or cytokines induced in the setting of parasitic infection, but not allergic disease may substitute for TL1A in costimulating ILC2. The exaggerated intestinal pathology, ILC expansion, and more rapid clearance of $N$. brasiliensis in DR3-deficient mice suggest that the absence of "signal 2" delivered through DR3 may sensitize ILC2 to cytokines induced during parasite infections that promote the host protective response. Group 2 ILC promote pathological allergic responses but provide benefit to the host through defense against intestinal parasites and tissue repair after influenza infection in the lung. ${ }^{44}$ Human counterparts of ILC2 have been found in the nasal passages of patients with allergic rhinitis ${ }^{45}$ and in the lung. ${ }^{44}$ Our findings suggest that blocking TL1A costimulation may reduce the contribution of ILC2-derived cytokines to allergic diseases while preserving the ability of these cells to function in host defense, an important goal in immunotherapy for allergic disease.

\section{METHODS}

Mice. C57BL/6 mice were obtained from Taconic (Hudson, NY). Tnfrsf $25^{-1-}$ mice were generated as previously described, ${ }^{46}$ and were backcrossed to the C57BL/6 background for at least 10 generations. TL1A Tg mice were produced as previously described ${ }^{16}$ and were backcrossed to the following mouse strains: Balbc.IL13R $\alpha 1^{-/-}$, $\mathrm{TCR} \alpha^{-1-}$ (Taconic), $\mathrm{Kit}^{\mathrm{W}-\mathrm{sH} / \mathrm{W}-\mathrm{sH}}, J \alpha 18^{-/-}, \mathrm{CD} 1 \mathrm{~d}^{-/-}, I l 13^{\text {eGFP } 26}$ 4C13R reporter mice. ${ }^{33}$

Histological quantification. Cross-sectional intestinal tissues were stained with Periodic Acid Schiff (PAS) or hematoxylin and eosin. Images were acquired with a BZ-9000 microscope (Keyence Corporation, Itasca, IL) and analyzed with the BZ-II Analyzer software package (Keyence Corporation). The mucosal thickness index was calculated by dividing the cross-sectional area of the muscularis and submucosa by the circumference of the center line of those tissues. Three sections were quantified per mouse. Average goblet cell area was calculated by measuring the average area of all goblet cells in five protruding villi in each mouse.

Antibiotic treatment and fecal DNA quantification. The antibiotics ampicillin $\left(1 \mathrm{gl}^{-1}\right)$, vancomycin $\left(500 \mathrm{mgl}^{-1}\right)$, neomycin trisulfate 
$\left(1 \mathrm{gl}^{-1}\right)$, and metronidazole $\left(1 \mathrm{gl}^{-1}\right)$ were added to the drinking water of cages housing mice from pregnancy or from birth. To harvest stool for DNA quantitation, the hindquarters and tails of mice were wiped with $70 \%$ ethanol and then held such that they defecated into sterile $2 \mathrm{ml}$ microcentrifuge tubes. Two pellets from each mouse were then processed with the QIAamp DNA Stool Mini Kit (Qiagen, Germantown, MD) according to the manufacturer's protocol and total DNA concentrations were quantified using a NanoDrop ND-1000 spectrophotometer (NanoDrop, Waltham, MA).

Cytokine measurement and analysis. IL-13 and Muc5ac mRNAs were measured by following the manufacturer's protocol with $100 \mathrm{ng}$ of total RNA, IL-25 and IL-33 mRNAs were measured with $200 \mathrm{ng}$ of total RNA using the ABI Taqman primer probe sets Mm00434204_m1, Mm01276718, Mm00499822_m1, and Mm00505403_m1, respectively, using BioRad iScript One Step RT-PCR reagents. Samples were run on a CFX96 RT-PCR detection system (Bio-Rad, Hercules, CA). For cytokine measurements by ex-vivo stimulated ILC, cells were cultured at 40,000 cells per well for $72 \mathrm{~h}$ and cytokine levels measured with the Millipore Magpix system (EMD Millipore, Billerica, MA).

IL-25, IL-33, and TL1A administration. In all, 0.4-2 $\mu$ g per dose of recombinant mouse IL-25, $0.5 \mu \mathrm{g}$ of recombinant mouse IL-33, or $5 \mu \mathrm{g}$ of TL1A (R\&D Systems, Minneapolis, MN) in phosphate-buffered saline (PBS) was administered daily for 3 or 4 days intraperitoneally. Mice were killed $24 \mathrm{~h}$ later and tissues harvested for analysis. Control animals received PBS only.

Mesenteric lymph nodes and lamina propria lymphocyte isolation. Cells from spleen and mesenteric lymph nodes were prepared by smashing the tissue through $40 \mu \mathrm{m}$ cell strainer. Lamina propria lymphocytes were extracted from the small intestine. In brief, the small intestine was collected and the Peyer's patches removed. Intestines were opened longitudinally and cut into small pieces $(<5 \mathrm{~mm})$. Intraepithelial cells were removed by washing with Hank's balanced salt solution and incubating with $5 \mathrm{mM}$ EDTA for $20 \mathrm{~min}$ at $37^{\circ} \mathrm{C}$. The intestinal pieces were washed with RPMI, and lamina propria lymphocytes were isolated by digestion with $1 \mu \mathrm{g} \mathrm{ml}^{-1}$ DNase (Sigma-Aldrich, St Louis, MO) and $500 \mu \mathrm{g} \mathrm{ml}^{-1}$ Collagenase D (Roche, Indianapolis, IN) for $30 \mathrm{~min}$ at $37^{\circ} \mathrm{C}$.

Parasite infection. Mouse-adapted N. brasiliensis were provided by Dr Joseph Urban (U.S. Dept. of Agriculture, Beltsville, MD) and their life cycle maintained as described. ${ }^{47}$ Mice were infected with $300-500$ L3 stage larvae by subcutaneous injection. Serum, perfused lungs, mesenteric lymph nodes, and rinsed intestines were collected at euthanasia. For histology, duodenum sections were slit lengthwise, rolled, and fixed in Bouin's Hollande. Adult worms were recovered from the entire small intestine by washing and counted using a dissection microscope. Tissue samples for RNA were collected from matched lung lobes or 2-4 equivalent samples from a grossly inflamed section of duodenum. For live egg counts, stool samples were collected daily, weighed, and dissolved in aqueous saturated $\mathrm{NaCl}$. Buoyant eggs were counted using a McMaster slide (Chalex, Grasonville, MD). S. mansoni were provided by the Biomedical Research Institute (Rockville, MD). Mice were infected with 35 cercariae by tail immersion as described ${ }^{48}$ and analyzed at 9 weeks. Adult worms were perfused from the mesenteric veins and counted. For RNA samples, tissue snips were collected from matched sites of three liver lobes and two to four sites of the small intestine. Histology specimens were fixed in Bouin's Hollande, stained with Giemsa, and evaluated by an experienced pathologist. The remaining liver tissue was dissolved in aqueous $4 \% \mathrm{KOH}$ and eggs were counted using a McMaster slide. Serum IL-13 and IL13R $\alpha 2$ was assayed as previously described. ${ }^{49}$

Ova-Induced lung inflammation. On days 0 and 7 , mice were sensitized systemically via a $200-\mu$ intraperitoneal injection containing either $100 \mu \mathrm{g}$ Chicken Ova (Sigma, St Louis, MO) or PBS emulsified in an equal volume mixture with alum (Thermo Scientific, Waltham, MA). For assessment of pulmonary inflammation, mice were challenged with $100 \mu \mathrm{g}$ Ova or PBS per $30 \mu \mathrm{l}$ inoculum intratracheally on day 14 and intranasally on day 15. Mice were euthanized $72 \mathrm{~h}$ after the final challenge. Bronchoalveolar lavage (BAL) fluid was obtained by direct cannulation of the lungs with a 20-gauge intravenous catheter and lavage with $500 \mu 11 \%$ fetal bovine serum in PBS (for cytokine analysis) and with $700 \mu \mathrm{l} \%$ fetal bovine serum in PBS (for analysis of cellular infiltration). Lung cell preps were obtained by incubating lung fragments with $100 \mathrm{U}$ collagenase for $1 \mathrm{~h}$. Cells were stained for surface antigens and intracellular cytokines after stimulation with phorbol 12-myristate 13-acetate/ionomycin for $4 \mathrm{~h}$. Hematoxylin and eosin and PAS-stained histological sections were scored by an observer masked to treatment group for peribronchiolar and perivascular cuffing (0-4), goblet cell hyperplasia (0-4), and interstitial infiltrate $(0-3)$.

Induction of papain-induced airway inflammation. Mice were anesthetized with isoflurane and exposed intranasally to $25 \mu \mathrm{g}$ papain (Calbiochem, Billerica, MA) in $30 \mu \mathrm{L}$ PBS on days 0,2 , 4, and 6 . In all, $12-16 \mathrm{~h}$ after the last challenge BAL was performed as described above. Lung isolated cells were obtained by incubating lung fragments with $100 \mathrm{U}$ collagenase for $1 \mathrm{~h}$. Cells were stained for surface antigens and intracellular cytokines after stimulation with phorbol 12-myristate 13acetate/ionomycin for $4 \mathrm{~h}$. Cells from the BAL were stained using antibodies against CD45, SiglecF, F4/80, Ly6G, and CD11b. Eosinophils were identified as $\mathrm{CD} 45^{+} \mathrm{F} 4 / 80^{-} \mathrm{Ly} 6 \mathrm{G}^{-} \mathrm{CD}_{11 \mathrm{~b}}{ }^{+}$SiglecF $^{+}$.

SUPPLEMENTARY MATERIAL is linked to the online version of the paper at http://www.nature.com/mi

\section{ACKNOWLEDGMENTS}

We would like to thank Masaki Terabe and Jay Berzofzky (NCl, NIH) for providing $\mathrm{J} \alpha 18$ and CD1d knockout mouse lines, Juan Rivera for providing W/WV mice, Anthony Cruz, Sarah Villareal, Odile Gabey, Nathan Bradley, and Allen Cheever for technical assistance, and Yasmine Belkaid and Aymen Al-Shamkhani for helpful discussions.

\section{DISCLOSURE}

Drs Siegel and Meylan have a patent application US 20120263718 related to $T L 1 A$ research.

c) 2014 Society for Mucosal Immunology

\section{REFERENCES}

1. Croft, M. The role of TNF superfamily members in T-cell function and diseases. Nat. Rev. Immunol. 9, 271-285 (2009).

2. Migone, T.S. et al. TL1A is a TNF-like ligand for DR3 and TR6/DcR3 and functions as a T cell costimulator. Immunity 16, 479-492 (2002).

3. Prehn, J.L., Thomas, L.S., Landers, C.J., Yu, Q.T., Michelsen, K.S. \& Targan, S.R. The T cell costimulator TL1A is induced by FcgammaR signaling in human monocytes and dendritic cells. J. Immunol. 178, 4033-4038 (2007).

4. Meylan, F. et al. The TNF-family receptor DR3 is essential for diverse T cell-mediated inflammatory diseases. Immunity 29, 79-89 (2008).

5. Meylan, F, Richard, A.C. \& Siegel, R.M. TL1A and DR3, a TNF family ligand-receptor pair that promotes lymphocyte costimulation, mucosal hyperplasia, and autoimmune inflammation. Immunol. Rev. 244, 188-196 (2011).

6. Pappu, B.P. et al. TL1A-DR3 interaction regulates Th17 cell function and Th17-mediated autoimmune disease. J. Exp. Med. 205, 1049-1062 (2008).

7. Bull, M.J. et al. The Death Receptor 3-TNF-like protein 1A pathway drives adverse bone pathology in inflammatory arthritis. J. Exp. Med. 205, 2457-2464 (2008).

8. Takedatsu, H. et al. TL1A (TNFSF15) regulates the development of chronic colitis by modulating both T-helper 1 and T-helper 17 activation. Gastroenterology 135, 552-567 (2008). 
9. Buchan, S.L., Taraban, V.Y., Slebioda, T.J., James, S, Cunningham, A.F. \& Al-Shamkhani, A. Death receptor 3 is essential for generating optimal protective CD4(+) T-cell immunity against Salmonella. Eur. J. Immunol. 42, 580-588 (2012).

10. Twohig, J.P. et al. The death receptor $3 / T L 1 A$ pathway is essential for efficient development of antiviral CD4(+) and CD8(+) T-cell immunity. FASEB J. 26, 3575-3586 (2012).

11. Bamias, G. et al. Expression, localization, and functional activity of TL1A, a novel Th1-polarizing cytokine in inflammatory bowel disease. J. Immunol. 171, 4868-4874 (2003)

12. Zhang, J. et al. Role of TL1A in the pathogenesis of rheumatoid arthritis. J. Immunol. 183, 5350-5357 (2009).

13. Bamias, G, Siakavellas, S.I., Stamatelopoulos, K.S., Chryssochoou, E, Papamichael, C \& Sfikakis, P.P. Circulating levels of TNF-like cytokine $1 \mathrm{~A}$ (TL1A) and its decoy receptor 3 (DcR3) in rheumatoid arthritis. Clin. Immunol. 129, 249-255 (2008).

14. Barrett, J.C. et al. Genome-wide association defines more than 30 distinct susceptibility loci for Crohn's disease. Nat. Genet. 40, 955-962 (2008).

15. Taraban, V.Y. et al. Sustained TL1A expression modulates effector and regulatory $T$-cell responses and drives intestinal goblet cell hyperplasia. Mucosal Immunol. 4, 186-196 (2011).

16. Meylan, F. et al. The TNF-family cytokine TL1A drives IL-13-dependent small intestinal inflammation. Mucosal Immunol. 4, 172-185 (2011).

17. Shih, D.Q. et al. Constitutive TL1A (TNFSF15) expression on lymphoid or myeloid cells leads to mild intestinal inflammation and fibrosis. PLOS ONE 6, e16090 (2011).

18. Twohig, J.P. et al. Age-dependent maintenance of motor control and corticostriatal innervation by death receptor 3. J. Neurosci. 30, 3782-3792 (2010).

19. McLaren, J.E. et al. The TNF-like protein 1A-death receptor 3 pathway promotes macrophage foam cell formation in vitro. J. Immunol. 184, 5827-5834 (2010).

20. Walker, J.A., Barlow, J.L. \& McKenzie, A.N. Innate lymphoid cells-how did we miss them?. Nat. Rev. Immunol. 13, 75-87 (2013).

21. Spits, H. et al. Innate lymphoid cells-a proposal for uniform nomenclature. Nat. Rev. Immunol. 13, 145-149 (2013).

22. Sawa, S. et al. Lineage relationship analysis of RORgammat + innate lymphoid cells. Science 330, 665-669 (2010).

23. Wong, S.H. et al. Transcription factor RORalpha is critical for nuocyte development. Nat. Immunol. 13, 229-236 (2012).

24. Yang, Q. et al. T cell factor 1 is required for group 2 innate lymphoid cell generation. Immunity 38, 694-704 (2013).

25. Moro, $\mathrm{K}$. et al. Innate production of $\mathrm{T}(\mathrm{H}) 2$ cytokines by adipose tissueassociated c-Kit $(+)$ Sca-1(+) lymphoid cells. Nature 463, 540-544 (2010).

26. Neill, D.R. et al. Nuocytes represent a new innate effector leukocyte that mediates type-2 immunity. Nature 464, 1367-U9 (2010).

27. Yang, Q, Saenz, S.A., Zlotoff, D.A., Artis, D \& Bhandoola, A. Cutting edge: natural helper cells derive from lymphoid progenitors. J. Immunol. 187, 5505-5509 (2011)

28. Maynard, C.L., Elson, C.O., Hatton, R.D. \& Weaver, C.T. Reciprocal interactions of the intestinal microbiota and immune system. Nature 489 , 231-241 (2012)

29. Hoyler, T. et al. The transcription factor GATA-3 controls cell fate and maintenance of type 2 innate lymphoid cells. Immunity 37 , 634-648 (2012).

30. Price, A.E. et al. Systemically dispersed innate IL-13-expressing cells in type 2 immunity. Proc. Natl. Acad. Sci. USA 107, 11489-11494 (2010).
31. Wilhelm, C. et al. An IL-9 fate reporter demonstrates the induction of an innate IL-9 response in lung inflammation. Nat. Immunol. 12, 1071-1077 (2011).

32. Barlow, J.L. et al. Innate IL-13-producing nuocytes arise during allergic lung inflammation and contribute to airways hyperreactivity. J. Allergy Clin. Immunol. 129, 191-198. e1-4 (2012).

33. Roediger, B. et al. Cutaneous immunosurveillance and regulation of inflammation by group 2 innate lymphoid cells. Nat. Immunol. 14, 564-573 (2013).

34. Hung, L.Y. et al. IL-33 drives biphasic IL-13 production for noncanonical Type 2 immunity against hookworms. Proc. Natl. Acad. Sci. USA 110, 282-287 (2013).

35. Fallon, P.G. et al. Identification of an interleukin (IL)-25-dependent cell population that provides IL-4, IL-5, and IL-13 at the onset of helminth expulsion. J. Exp. Med. 203, 1105-1116 (2006).

36. Chiaramonte, M.G., Donaldson, D.D., Cheever, A.W. \& Wynn, T.A. An IL-13 inhibitor blocks the development of hepatic fibrosis during a T-helper type 2-dominated inflammatory response. J. Clin. Invest. 104, 777-785 (1999).

37. Chiaramonte, M.G., Schopf, L.R., Neben, T.Y., Cheever, A.W., Donaldson, D.D. \& Wynn, T.A. IL-13 is a key regulatory cytokine for Th2 cell-mediated pulmonary granuloma formation and IgE responses induced by Schistosoma mansoni eggs. J. Immunol. 162, 920-930 (1999).

38. Sokol, C.L., Chu, N.-Q., Yu, S, Nish, S.A., Laufer, T.M. \& Medzhitov, R. Basophils function as antigen-presenting cells for an allergen-induced $T$ helper type 2 response. Nat. Immunol. 10, 713-720 (2009).

39. Tang, H. et al. The Thelper type 2 response to cysteine proteases requires dendritic cell-basophil cooperation via ROS-mediated signaling. Nat. Immunol. 11, 608-617 (2010).

40. Kamijo, S. et al. IL-33-mediated innate response and adaptive immune cells contribute to maximum responses of protease allergen-induced allergic airway inflammation. J. Immunol. 190, 4489-4499 (2013).

41. Pichery, M. et al. Endogenous IL-33 is highly expressed in mouse epithelial barrier tissues, lymphoid organs, brain, embryos, and inflamed tissues: in situ analysis using a novel II-33-LacZ gene trap reporter strain. J. Immunol. 188, 3488-3495 (2012).

42. Halim, T.Y., Krauss, R.H., Sun, A.C. \& Takei, F. Lung natural helper cells are a critical source of Th2 cell-type cytokines in protease allergen-induced airway inflammation. Immunity 36, 451-463 (2012).

43. Paul, W.E. \& Zhu, J. How are TH2-type immune responses initiated and amplified? Nat. Rev. Immunol. 10, 225-235 (2010).

44. Monticelli, L.A. et al. Innate lymphoid cells promote lung-tissue homeostasis after infection with influenza virus. Nat. Immunol. 12, 1045-1054 (2011).

45. Mjosberg, J.M. et al. Human IL-25- and IL-33-responsive type 2 innate lymphoid cells are defined by expression of CRTH2 and CD161. Nat. Immunol. 12, 1055-1062 (2011).

46. Wang, E.C.Y., Thern, A, Denzel, A, Kitson, J, Farrow, S.N. \& Owen, M.J. DR3 regulates negative selection during thymocyte development. Mol. Cell. Biol. 21, 3451 (2001).

47. Camberis, M, Le Gros, G \& Urban, J Jr Animal model of Nippostrongylus brasiliensis and Heligmosomoides polygyrus. Curr. Protoc. Immunol., Chapter 19:Unit 192 (2003)

48. Lewis, F. Schistosomiasis. Curr. Protoc. Immunol., Chapter 19:Unit 191 (2001).

49. Khodoun, M. etal. Differences in expression, affinity, and function of soluble (s)IL-4Ralpha and sIL-13Ralpha2 suggest opposite effects on allergic responses. J. Immunol. 179, 6429-6438 (2007). 